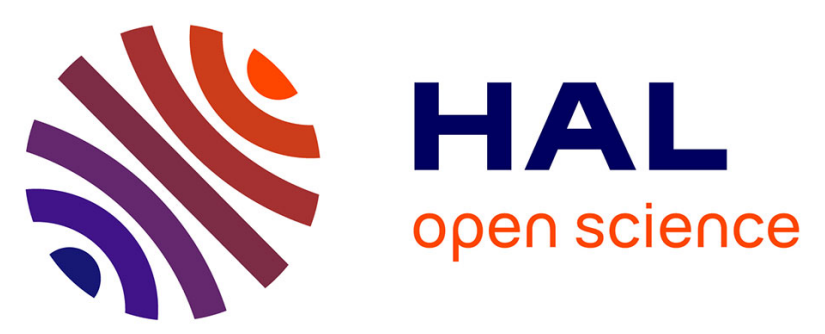

\title{
Suitability of a PLCL fibrous scaffold for soft tissue engineering applications: A combined biological and mechanical characterisation
}

Cédric P. Laurent, Cédryck Vaquette, Xing Liu, Jean-Francois Schmitt,

Rachid Rahouadj

\section{To cite this version:}

Cédric P. Laurent, Cédryck Vaquette, Xing Liu, Jean-Francois Schmitt, Rachid Rahouadj. Suitability of a PLCL fibrous scaffold for soft tissue engineering applications: A combined biological and mechanical characterisation. Journal of Biomaterials Applications, 2018, 32 (9), pp.1276-1288. 10.1177/0885328218757064. hal-02087521

\section{HAL Id: hal-02087521 \\ https://hal.univ-lorraine.fr/hal-02087521}

Submitted on 18 Apr 2019

HAL is a multi-disciplinary open access archive for the deposit and dissemination of scientific research documents, whether they are published or not. The documents may come from teaching and research institutions in France or abroad, or from public or private research centers.
L'archive ouverte pluridisciplinaire HAL, est destinée au dépôt et à la diffusion de documents scientifiques de niveau recherche, publiés ou non, émanant des établissements d'enseignement et de recherche français ou étrangers, des laboratoires publics ou privés. 
1 Article type

2 original manuscript

3 Corresponding author

4 Cédric LAURENT

5 LEMTA, 2 avenue de la forêt de Haye 54502 Vandoeuvre-lès-Nancy, France

60033383595581

7 cedric.laurent@univ-lorraine.fr

8

9 Suitability of a PLCL fibrous scaffold for

10 Soft tissue engineering applications:

11 a combined biological and mechanical

12 characterization

13 Cédric P. Laurent 1,*, Cédryck Vaquette ${ }^{2}$, Xing Liu ${ }^{3}$, Jean-

14 François Schmitt ${ }^{1}$, Rachid Rahouadj ${ }^{1}$

$15 *$ corresponding author.

$16{ }^{1}$ CNRS, LEMTA, UMR 7563, Université de Lorraine, 2 avenue de la forêt de

17 Haye 54502 Vandoeuvre-lès-Nancy, France.

$18{ }^{2}$ Queensland University of Technology (QUT), Brisbane, Australia

$19{ }^{3}$ CNRS, IMoPA, UMR 7365, Biopôle, Université de Lorraine, 9 Avenue de la Forêt de

20 Haye, 54504 Vandeuvre-lès-Nancy, France

21 Keywords

22 PLCL ; fibrous scaffold; ligament tissue engineering; stem cells ; degradation ;

23 biocompatibility 


\section{1 a combined biological and mechanical 2 characterization}

3 Abstract

4 Poly(lactide-co- $\varepsilon$-caprolactone) PLCL has been reported to be a good candidate for tissue 5 engineering, based on its good biocompatibility. Particularly, a braided PLCL scaffold $6 \quad(\mathrm{PLL} / \mathrm{PCL}$ ratio $=85 / 15)$ has been recently designed and partially validated for ligament 7 tissue engineering. In the present study, we assessed the in vivo biocompatibility of 8 acellular and cellularized scaffolds in a rat model. We then determined its in vitro 9 biocompatibility using stem cells issued from both bone marrow and Wharton Jelly.

10 From a biological point of view, the scaffold was shown to be suitable for tissue 11 engineering in all these cases. Secondly, while the initial mechanical properties of this 12 scaffold have been previously reported to be adapted to load-bearing applications, we 13 studied the evolution in time of the mechanical properties of PLCL fibers due to 14 hydrolytic degradation. Results for isolated PLCL fibers were extrapolated to the fibrous 15 scaffold using a previously developed numerical model. It was shown that no 16 accumulation of plastic strain was to be expected for a load-bearing application such as 17 anterior cruciate ligament tissue engineering. However, PLCL fibers exhibited a non18 expected brittle behavior after two months. This may involve a potential risk of 19 premature failure of the scaffold, unless tissue growth compensates this change in 20 mechanical properties. This combined study emphasizes the need to characterize the 21 properties of biomaterials in a pluridisciplinary approach, since biological and 22 mechanical characterizations led in this case to different conclusions concerning the 23 suitability of this scaffold for load-bearing applications.

\section{Keywords}

25 PLCL ; fibrous scaffold; ligament tissue engineering; stem cells ; degradation ; 26 biocompatibility 
Among the variety of biodegradable polymers commonly used in tissue engineering (see 3 for instance recent reviews such as ${ }^{1-8}$ ), poly(lactide-co- $\varepsilon$-caprolactone) PLCL has been 4 reported to be a good candidate for tissue engineering. Indeed, the association of polylactic acid (PLLA) with poly(e-caprolactone) (PCL) into PLCL has been reported to compensate both the brittle behaviour of PLLA and the low stiffness of PCL ${ }^{9,10}$ as well as the local acidification due to the degradation of PLLA ${ }^{11}$. It also offers a slow degradation rate adapted to its use in tissue engineering, tailorable by changing the PCL/PLLA ratio ${ }^{12}$. A strong shape-memory effect has also been observed for PLCL, depending on PCL/PLLA ratio ${ }^{13}$. The biocompatibility of PLCL in various shapes such as foams ${ }^{14}$, sheet-form scaffolds ${ }^{15}$, tubular porous scaffolds ${ }^{16-18}$, electrospun microfibers ${ }^{19-24}$ and tubular electrospun scaffolds ${ }^{25}$ has been confirmed. PLCL in these various forms has been recently treated by plasma ${ }^{24,26}$, loaded with heparin ${ }^{27,28}$ of growth factors $^{29}$, or blended with gelatin ${ }^{20,30,31}$ to improve cellular response. From these different assessments, it may be concluded that PLCL is a suitable material for various tissue engineering applications, especially when "elasticity and degradability are required in the same product" 10 .

Among the range of these various applications, PLCL has been used for ligament tissue engineering applications in our group ${ }^{32-35}$ or others ${ }^{36}$. Indeed, we have recently developed a braided PLCL scaffold for ligament tissue engineering with promising preliminary results concerning its mechanical, morphological and biological properties ${ }^{32-}$ 34. Particularly, this scaffold has been shown to be adapted to a computer-aided tissue engineering approach ${ }^{37}$, helping to predict and optimize both the scaffold properties and the cell's micro-environment that controls cellular activity. This geometry and the associated equipment developed for its processing could easily be applied to other clinical applications such as arteries, nerves or tendons. From a purely biological point of view, in vivo studies reporting the results of the implantation of such PLCL scaffolds are still lacking in the literature in order to assess their biological suitability, and in vitro studies for different culture conditions still need to be performed. The first objective of the present contribution is therefore to provide additional data concerning the biological suitability of PLCL braided scaffolds based on results of in vivo and in vitro experiments. Particularly, these in vivo and in vitro studies respectively focus on (1) the effect of initial cellularisation of the scaffold on its capability to be colonized and vascularized in vivo (2) the effect of cell source on the colonization of the scaffold. Indeed, for this second point, current reported ${ }^{34}$ studies on this PLCL scaffold have used stem cells issued from bone marrow, while other types of cells such as Wharton-jelly stem cells are gaining attention 38 .

Despite these biological advantages of PLCL for tissue engineering applications, it has been reported that PLCL may be subject to ageing, and that post-crystallisation may have a drastic effect on its mechanical properties ${ }^{10,39,40}$. The estimation of scaffold degradation has often been limited to mass $\operatorname{loss}^{41}$, not necessarily linked to mechanical properties. When tested, the evolution in time of mechanical properties have been restricted to apparent properties (compressive modulus, ultimate strength, ...) and limited to the elastic range ${ }^{42,43}$. The effect of hydrolytic degradation on the mechanical behaviour of 
PLCL scaffolds has been insufficiently reported, while this constitutes a milestone in tissue engineering ${ }^{44}$. Indeed, this evolutive behavior should be controlled since (1) the scaffold should fulfil the physiological function of the native tissue during the postsurgery phase (2) accumulated plastic strain within the scaffold may lead to unsuited graft laxity for load-bearing applications (3) the potential loss of mechanical properties in this period due to scaffold degradation should be compensated by tissue growth. The question of how these results can be confronted and used to assess the suitability of a scaffold for a given clinical application is then crucial. Indeed, the characterized initial mechanical properties may not be extrapolated to the post-implantation phase. Consequently, despite the encouraging reported studies concerning the biological suitability of PLCL for soft tissue engineering, data concerning the evolution of its mechanical properties during degradation are still lacking. This gap in knowledge may be partially explained by the extent of the campaign that should be carried on in order to perform the required mechanical experiments. A solution consists in chemically accelerating the hydrolysis, which has been recently applied to study the complex degradation mechanisms of a PCL scaffold for tendon tissue engineering ${ }^{44}$. Alternatively,

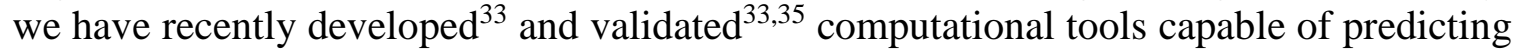
the mechanical properties of braided scaffolds from the properties of isolated fibres. Such computational tools may drastically reduce the extent of the experimental campaign. These tools may incorporate recent models based on damage ${ }^{45-48}$ developed in order to take into account the variation of polymer mechanical properties during hydrolytic degradation. The second objective of the present contribution is therefore to study the change of mechanical properties of PLCL during degradation. This mechanistic point of view concerning the suitability of PLCL for soft tissue engineering applications brings information to be confronted to the reported biological results.

In the present paper, a braided scaffold made of PLCL in a PLA/PCL ratio of 85/15 is used. This particular polymer has been selected previously ${ }^{32}$ based on a trade-off between deformability and stiffness. In a first section, the results of in vivo implantations of acellular and cellularized scaffolds after 1 and 4 weeks in a rat model are presented. This is completed with results of in vitro culture for two different types of stem cells. Conclusions are thus drawn concerning the biocompatibility of the PLCL scaffold from these combined in vivo and in vitro studies. In a second part, the effect of hydrolytic degradation on the mechanical properties of PLCL fibres is assessed, and extrapolated to the braided scaffold using a computational model. Some new conclusions are then provided and discussed concerning both the biological and mechanical suitability of this polymer for soft tissue engineering applications.

Material and Methods

Processing of PLCL fibres and scaffold preparation

39 The braided scaffold used in this study has already been described previously ${ }^{32,33}$. 40 Briefly, it consisted of an assembly of 6 layers of 16 fibres each (96 fibres), arranged into 41 a circular braid. Fibres were made of PLCL with a PLLA/PCL ratio of 85/15 (Corbion 42 Purac biomaterials, The Netherlands). Fibres were first processed into $180 \mu \mathrm{m}$ fibres 
1 using a dedicated plastic extruder ${ }^{32}$, and then arranged into concentric circular braids

2 using a Maypole braiding machine (Composite \& Wire machinery, United States).

\section{In vitro assessment of biocompatibility}

4 Mesenchymal Stem Cells isolation and expansion.

5 The suitability of both Bone Marrow Mesenchymal Stem Cells (BM-MSC) and Wharton Jelly Mesenchymal Stem Cells (WJ-MSC) have been reported to be comparable for tissue engineering applications ${ }^{38}$. In this in vitro study, these two types of cells were therefore 8 used and confronted. Human bone marrow and human umbilical cords were harvested and supplied by CHRU Hospital and Maternity Hospital (Nancy, France) respectively with the informed consent of patients. For BM-MSC, 25mL $\alpha$-MEM (Lonza, BE12169F) medium contained 10\%FBS (Dominique Dutscher), $2 \mathrm{mM} \mathrm{L-glutamine} \mathrm{(Sigma,} \mathrm{A-}$ 4034), $100 \mathrm{U} / \mathrm{mL}$ penicillin /streptomycin (Gibco, 15140-122) and $1 \mu \mathrm{g} / \mathrm{mL}$ amphoterin B (Gibco, 15290-026) was added to $20 \mathrm{~mL}$ of bone marrow, and centrifuged $300 \mathrm{~g}$ for $5 \mathrm{~min}$. The supernatant was removed, cells were counted to be seeded on culture flask at the density of 50000 cells $/ \mathrm{cm} 2$, then incubated in $37^{\circ} \mathrm{C}, 5 \% \mathrm{CO}_{2}, 90 \%$ humidity. The culture medium was changed twice a week. For WJ-MSC, human umbilical cord was first washed with $70 \%$ ethanol and cut into $3-4 \mathrm{~cm}$ in the HBSS buffer. The cord was opened and wharton-jelly was exposed, and the jelly was torn off from cordon and cut into fine pieces, and transferred to 6-well plates. After 7 days, the jelly was removed, the culture medium was changed twice a week. All MSC used for assays are in passage 2(P2).

MSC metabolic activity and morphology on scaffold.

The scaffolds were washed with PBS, then exposed to UV for 15 min each side. MSC with density of 300,000 cells $(0.05 \mathrm{~mL}$ were distributed on each scaffold. MSCscaffolds were then put in the incubator for $30 \mathrm{~min}$, and supplied with $0.95 \mathrm{~mL} \alpha$ - MEM complete medium with ascorbic acid (Sigma, A-4034). Cells were cultured on the scaffolds for 2 weeks. Alamar Blue (AB) tests were performed on D1, D3, D5, and D7. $1 \mathrm{~mL} 10 \% \mathrm{AB}$ work solution (Thermo scientific, 88952) was added to each scaffold, and incubated for $3 \mathrm{~h}$ at $37{ }^{\circ} \mathrm{C}$, the solution was then aspired to test the optical density at $570 \mathrm{~nm}$ and $630 \mathrm{~nm}$. Each well was repeated 3 times. The negative control was $A B$ working solution without cells. MSC-Scaffolds were first fixed with $1 \%$ paraformaldehyde for 10 minutes at room temperature then washed 3 times with PBS. 1 $\mathrm{mL} 0.5 \%$ triton-PBS was added to each for 20 minutes at room temperature. Scaffolds were washed 3 times with $1 \mathrm{~mL}$ PBS. $250 \mu \mathrm{L}$ Alexa 488 Phalloidin solution was added to each scaffold for 45 minutes. Scaffolds were then washed 3 times with PBS. $200 \mu \mathrm{L}$ DAPI (1/1500) was added to each scaffold for $15 \mathrm{~min}$. Scaffold were washed 3 times again with PBS and observed by florescence microscopy. Wavelengths used for florescence microscopy were respectively $456 \mathrm{~nm}$ for DAPI and 509nm for Alea 488 phalloidin.

39 Collagen synthesized on scaffold by immunofluorescence staining.

40 Firstly, MSC-scaffolds were fixed with $1 \%$ paraformaldehyde for 10 minutes at room 41 temperature, and washed 3 times with PBS. Then, 5\% PBS-BSA blocking buffer was 42 added to scaffolds for $1 \mathrm{~h}$ at room temperature, then washed 3 times with PBS. $250 \mu \mathrm{L}$ of 
1 the primer antibody collagen I rabbit (CALBIOCHEM, 234167), collagen III mouse 2 (Sigma, C7805) at the dilution of $1 / 50$, were added to scaffold for $1 \mathrm{~h}$ at room 3 temperature, then washed 3 times with PBS. The second antibody Anti-Rabbit IgG4 Alexa488 (1/50) (life technologies, A11008) and Anti-mouse IgG-Alexa488 were added $5 \quad(250 \mu \mathrm{L})$ to scaffold for 45 mins, washed 3 times with PBS. $250 \mu \mathrm{L}$ DAPI $(1 / 1500)$ was 6 added to each scaffold for $15 \mathrm{mins}$, and kept isolated from light. MSC-Scaffolds were washed 3 times with PBS, and imaged with fluorescence microscopy.

\section{In vivo implantation of scaffolds}

The in vivo behaviour of PLCL braided scaffolds towards tissue colonisation and neovascularization was assessed using a subcutaneous implantation site in an immunocompromised rodent model. The experiment also compared the regenerative performance of cell seeded scaffolds to non-cellularised constructs and evaluated the impact of bone marrow mesenchymal stem cell on tissue remodelling. Scaffolds were sterilised by immersion in $70 \%$ ethanol for 30 min and subsequently UV-irradiated for 30 min and finally dried overnight in a biosafety cabinet as previously reported ${ }^{34}$. Human BM-MSC (Tulane University Center for Gene Therapy, USA) were expanded in DMEM supplemented with $16.5 \%$ calf foetal serum and $1 \%(\mathrm{v} / \mathrm{v})$ antibiotics cocktail (Merckmillipore, 10,000 U/mL penicillin G, $10 \mathrm{mg} / \mathrm{mL}$ streptomycin, $25 \mu \mathrm{g} / \mathrm{mL}$ amphotericin B) as per the supplier protocol until $80 \%$ confluency. They were seeded into the braided scaffold (10 mm long) at P5 with a cell seeding density of 500,000 cells per scaffold. The seeding was performed in two steps to ensure homogeneous seeding: 250,000 cells in $50 \mu \mathrm{L}$ of media were added onto the structure which was subsequently turned over to receive another 250,000 cells in $50 \mu$. The samples were placed in the incubator and $20 \mu \mathrm{L}$ of warm media was added every $15 \mathrm{~min}$ for $2 \mathrm{~h}$ and subsequently every $30 \mathrm{~min}$ for another $2 \mathrm{~h}$. At the end of the 4 hours incubation period, the wells were filled with $1 \mathrm{~mL}$ of warm media supplemented with $100 \mu \mathrm{g} / \mathrm{mL}$ of ascorbate-2-phosphate to stimulate collagen production. The braided scaffolds were cultured under static conditions for 4 weeks with a 3 -weekly media change.

Animal ethics approval for the use of athymic nude rats in this experiment was granted by the Animal Ethics Committee of Griffith University. Six 8-week old male rats (Sprague-Dawley outbred rat model, Animal Resources Centre, Canning Vale, WA, Australia) were used. The animals were anaesthetized with isoflurane. Four small incisions were made $2 \mathrm{~cm}$ apart from the central line of the shaved dorsal area and subcutaneous pockets were using a pair of surgical scissors. In each animal, 2 scaffolds and 2 cellularised scaffolds were implanted. Each individual pocket held one scaffold and the incisions were closed with surgical sutures. The animals were sacrificed after 1 or 4 weeks post-implantation. The implants were retrieved and fixed in $4 \%$ paralformaldehyde in PBS at $\mathrm{pH} 7.4$ for $24 \mathrm{~h}$ and thereafter immersed in PBS until further analysis. The samples were subsequently embedded in paraffin, sections near the central area of the specimens were used for haematoxylin and eosin (H\&E) staining. The slides were scanned using the slide scanner Scanscope (Leica). 
Tensile tests were performed using a Zwick Roell 2.5 device (Zwick, Germany) equipped with a $2.5 \mathrm{kN}$ cell force, at a tensile speed of $0.1 \mathrm{~mm} . \mathrm{s}^{-1}$. Tests were performed in water with controlled temperature. $60 \mathrm{~cm}$-long fibres were rolled up and then fixed around cylindrical parts (see Figure 1(a)), in such a way that 6 fibres were tested in parallel. This setup was designed to (1) limit stress concentration at the level of fibre gripping (2) increase the sensitivity of force measurement (3) average the measurements of fibre properties by using several fibres. At each time step, four tensile tests were carried out, and the second Piola-Kirchhoff stress $S_{11}$ and the Green-Lagrange strain $E_{11}$ were 9 computed from measured displacements and forces (where index 1 stands for the loading 10 direction). This formulation is compatible with the large strain framework in which these 11 tests were performed. The following loading cycle was prescribed (1) $1 \%$ strain then unloading (2) $3 \%$ strain then unloading (3) increasing strain up to failure (see Figure 1(b)). The typical stress-strain response of PLCL polymer fibers at the initial time is 14 represented on Figure 1(c).

15

(a)

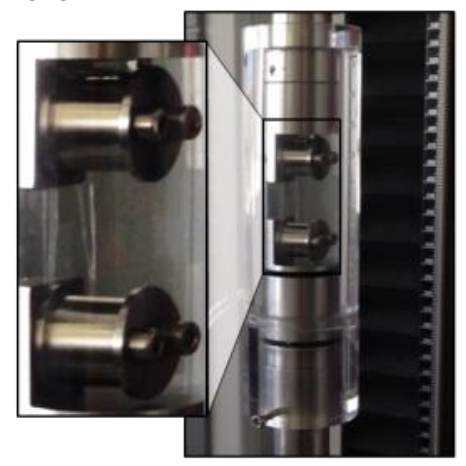

(b)

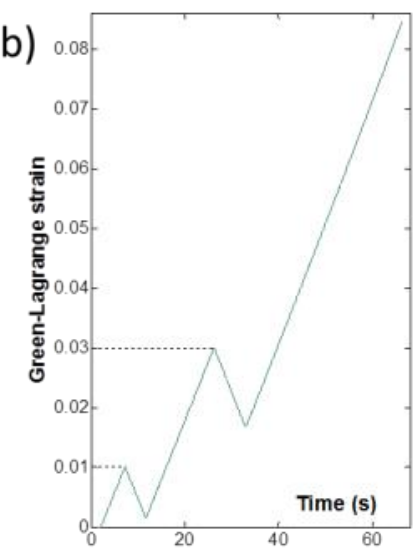

(c)

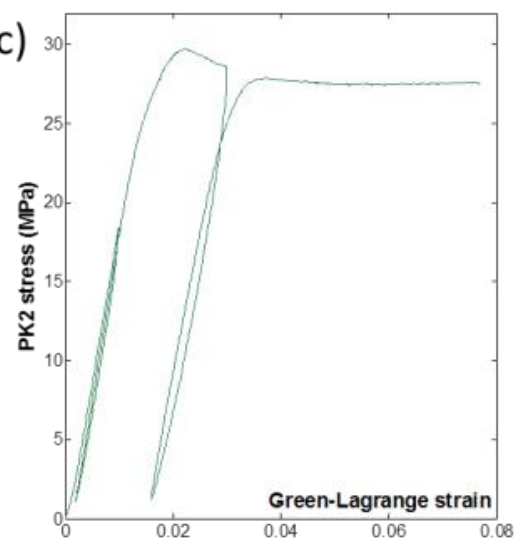

Figure $1:$ (a) Design of the setup used to evaluate uniaxial tensile properties of isolated polymer fibres. (b) Loading path prescribed to the set of fibres. (c) Typical stress-strain response of set of fibres before hydrolytic degradation (day 0 ).

\section{Numerical simulations for braided PLCL scaffold}

The FE code dedicated to fibrous material used in this study has been previously described elsewhere ${ }^{49}$, as well as its application to the braided polymer scaffold ${ }^{33,35}$. Briefly, in the simulations, each fibre was as a kinematically enriched finite strain beam model accounting for cross-sectional strains within a finite geometrical transformation framework. An original procedure was then used to detect the numerous contacts within the assembly of fibres, based on intermediate geometries used to create discrete contact elements and to determine the normal directions to the contact areas. The FE code predicted the initial geometry of the braid (and the associated initial stresses) from an arbitrary reference configuration in which fibres interpenetrated each other ${ }^{35}$. The noninterpenetrated configuration was then gradually computed from the knowledge of the 
braiding pattern within each scaffold layer. Average boundary conditions were defined on each scaffold layer using rigid bodies, thus enabling transverse rearrangement of individual fibres. Results were finally exported in terms of scaffold geometry at each loading step, strain tensors at many points generated on the surface of individual fibres, and global forces and moments. Both the predicted response of the scaffold ${ }^{33}$ and the predicted geometries of the collection of fibres ${ }^{35}$ have been previously validated. In the FE code, the three-dimensional elasto-plastic response of isolated fibres was modelled using the relation:

$$
\left\{\begin{array}{lll}
\mathbf{S}=\mathbf{C}: \mathbf{E} & \text { if } & E_{11}<\varepsilon_{0} \\
\mathbf{S}=\mathbf{C}: \mathbf{E}_{e l}+Y\left(E_{11}\right) \mathbf{C}:\left(\mathbf{E}-\mathbf{E}_{e l}\right) & \text { if } & E_{11} \geq \varepsilon_{0}
\end{array}\right.
$$

9 where $\mathbf{S}$ is the stress tensor, $\mathbf{E}$ is the strain tensor, $\mathbf{C}$ is the elastic stiffness tensor, $Y$ is a 10 yield function depending on the axial strain $E_{11}, \mathbf{E}_{e l}$ is the elastic part of the deformation, 11 and $\varepsilon_{0}$ is the yield strain. The yield function $Y$ was expressed as follows ${ }^{50}$ :

$$
Y\left(E_{11}\right)=a+b\left(1-e^{-c E_{11}}\right)
$$

12 where $a, b, c$ are three material constants. The continuity of $S_{11}$ and $\partial S_{11} / \partial E_{11}$ at $E_{11}=\varepsilon_{0}$ 13 gives conditions on these constants, and consequently:

$Y\left(E_{11}\right)=E \varepsilon_{0}-\left(\frac{E}{c e^{-c \varepsilon_{0}}}\right)\left(e^{-c E_{11}}-e^{-c \varepsilon_{0}}\right)$

14 The elasto-plastic response of fibres was thus fully described by 3 material parameters: the elastic modulus $E$, the yield strain $\varepsilon_{0}$ and a hardening parameter $c$.

16 In the performed simulations, the case of Anterior Cruciate Ligament (ACL) was considered as an application of the braided scaffold within a given physiological environment. From the computed initial geometry, a pretension phase was first simulated in order to condition the scaffold, as commonly done in clinics ${ }^{51}$. This phase consisted of a prescribed global strain of $4 \%$, followed by a total unloading. Secondly, in order to evaluate the response of the braided scaffold to physiological loads, boundary conditions were defined such as they mimicked squats (i.e. one of the most common rehabilitation activities) : this was done by prescribing a $3.6 \%$ axial strain ${ }^{52}$. Simulated force-strain curves for each time step were thus collected. The FE code was also used to determine of the Octahedral Shear Strain (OSS) at the surface of scaffold fibres. This stimulus was considered here to be the most influencing mechanical signal on cellular response as suggested in the literature ${ }^{53}$. OSS is defined as follow:

$$
O S S=\frac{2}{3} \sqrt{\left(\varepsilon_{I}-\varepsilon_{I I}\right)^{2}+\left(\varepsilon_{I I}-\varepsilon_{I I I}\right)^{2}+\left(\varepsilon_{I I I}-\varepsilon_{I}\right)^{2}}
$$

where $\varepsilon_{I}, \varepsilon_{I I}, \varepsilon_{I I I}$ stand for the principal strains of the fibre scale Green-Lagrange strain tensor. The evolution of OSS during a squat exercise was investigated. OSS was set to zero at the end of the loading-unloading pretension phase, and then the distribution of OSS at the scaffold's surface at the end of the simulated exercise (strain $=3.6 \%$ ) were analysed. 


\section{Effect of hydrolytic degradation on mechanical properties}

3 Tensile tests were performed on isolated PLCL fibres, at given times after the extrusion 4 process. Prior to the extrusion, raw polymer was stored at $-80^{\circ} \mathrm{C}$. After the extrusion, 5 polymer fibres were maintained in an incubator at $37^{\circ} \mathrm{C}$ within distilled water, and tested 6 in uniaxial tension at dedicated time points (day 0, 5, 8, 14, 19, 26, 32, 40, 49, 54, 60, 67, 7 75). In recent studies $45,46,48$, it has been proposed that hydrolytic degradation may be 8 modeled using a damage variable expressed as :

$d=1-e^{-\mu t} \quad 0 \leq d \leq 1$

9 where $t$ is the time and $\mu$ the strength decrease rate. Based on this work, we modelled

10 the evolution of the Young's modulus of fibres using a damage parameter following an

11 exponential law, such that the Young's modulus is nul after full degradation :

$$
E(t)=E_{0}\left(1-d^{E}(t)\right) \quad \text { with } \quad d^{E}(t)=1-e^{-\mu^{E} t}
$$

12 In this equation, $E_{0}$ is the initial Young's modulus and $\mu^{E}$ was fitted from experiments.

13 Nonetheless, to the best of our knowledge, there was no fundamental basis enabling us to 14 formulate the evolution of the yield strain $\varepsilon_{0}$ and the hardening parameter $c$ as a function

15 of the degradation: therefore these laws were based on the results of our experiments, and 16 formulated in a purely phenomenological way. In order to fit the Chaboche-type elasto17 plastic model to experimental data, a least-square minimization method was used based 18 on the first part of the stress-strain curve, i.e. until the maximal stress is reached, ignoring 19 the first unloading cycle.

\section{Results}

\section{In vitro assessment of biocompatibility}

22 WJ-MSC and BM-MSC were seeded on the scaffold, and the cell metabolic ability under 23 static culture was detected by Alamar Blue. For WJ-MSC and BM-MSC, cell metabolic 24 ability both showed increased trend during one week (Figure 2). For BM-MSC, the 25 metabolic activity increased from $50 \%$ in D1 to $71.1 \%$ in D7, while for WJ-MSC, it 26 increased from $37 \%$ in D1 to $63.7 \%$ in D7. In day14, cells were fixed and cell skeleton (green) and cell nuclei(blue) were stained to show the cell morphology and cell location on the scaffold. Cells proliferated well on PLCL scaffold, and bridges were formed

29 between fibers, after one week. 

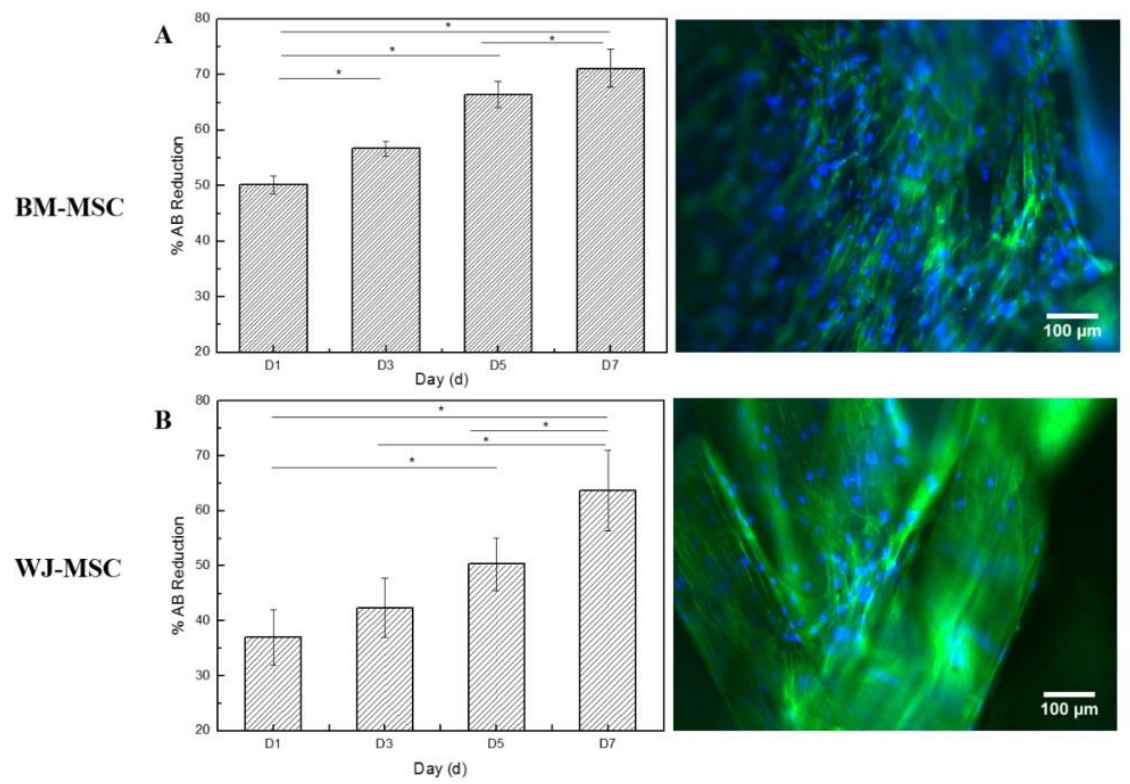

2 Figure 2: (left) $\mathrm{AB}$ reduction of $\mathrm{BM}-\mathrm{MSC}(\mathrm{A})$ and $\mathrm{WJ}-\mathrm{MSC}(\mathrm{B})(* \mathrm{p}<0.05)$ and (right) 3 immunofluorescence staining of WJ-MSC and BM-MSC on scaffolds. (cell skeleton 4 (green, 509nm) and cell nuclei (blue, 456nm)).

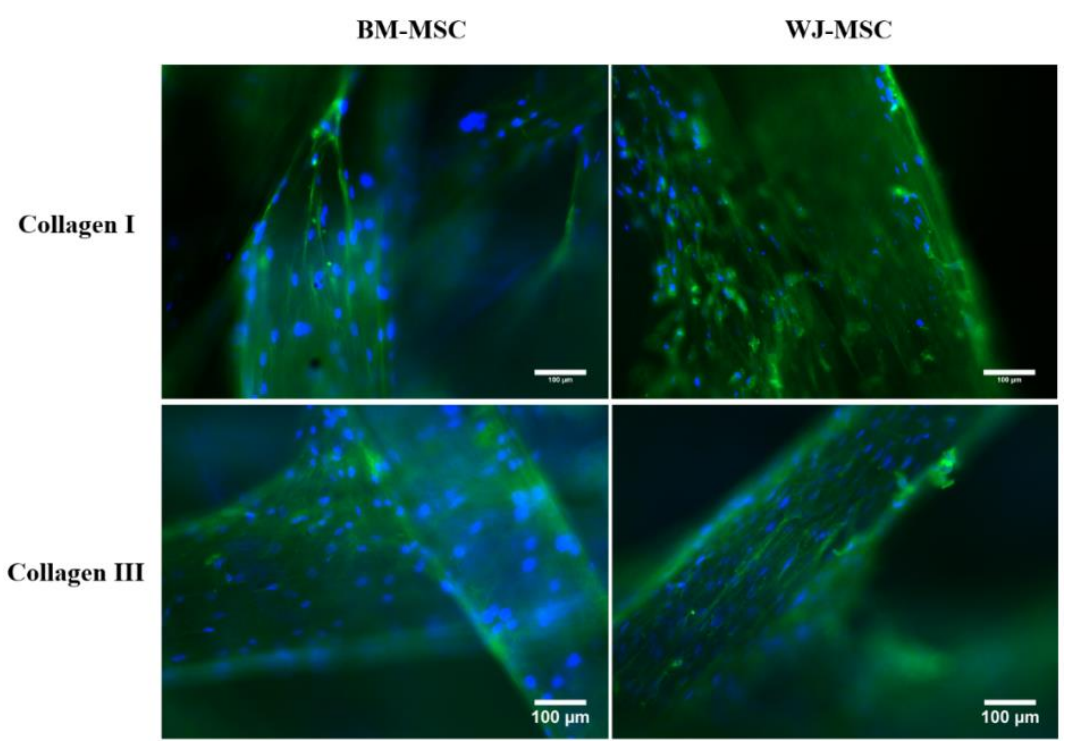

6 Figure 3: Collagen I and collagen III expression by WJ-MSC and BM-MSC on scaffolds 7 in D14. (collagen (green, 509nm) and cell nuclei (blue, 456nm))

8 The expression of collagen secreted by BM-MSC and WJ-MSC on PLCL scaffolds was 9 detected by immunofluorescence staining. As showed in Figure 3, collagen I and III were 10 synthesized by BM-MSC and WJ-MSC on the scaffolds. Collagen (greed dot on Figure

11 3) was found to cover the fibers and form bridges between them. No significant 12 differences were observed between BM-MSC and WJ-MSC. 


\section{In vivo implantation of scaffolds}

2 The healing course of the animals was uneventful and Figure 4 displays the 3 representative gross morphology of the retrieved samples at 1and 4 weeks. After 1 week 4 post-implantation the braided scaffold integrated well with the surrounding tissue, a 5 fibrous capsule was formed around the implant and the presence of numerous blood 6 vessels in the direct vicinity of the scaffolds (square arrow head Figure 4 A and B) was 7 noted regardless of the group. A red colour was also observed in the core of the braided 8 scaffold suggesting high vascularisation. Similar observations were made 4 weeks post-

9 implantation, with the presence of blood vessels in the direct proximity of the scaffold 10 (square arrow head).

11 The H\&E staining revealed that the scaffolds were fully colonised by the host tissue as 12 early as 1 week post-implantation as shown in Figure $4 \mathrm{~A}$ and B regardless of the 13 presence of MSC. In both cases, a dense collagenic network had formed within the pore 14 of the braided structure. A mild inflammatory response was also observed on the 15 periphery of the filaments (indicated by the *, note that the polymer was in some location 16 dissolved during the histological sample processing hence leaving a void) with 17 polynucleated cells (round arrow head) surrounding the polymeric material. The tissue in 18 the central portion of the braided scaffolds was particularly well vascularised with the 19 presence of numerous microvessels but also larger blood vessels (black arrows) for both 20 acellular and cellularised constructs.

21 Similar features were observed at 4 week post-implantation demonstrating that the scaffold was capable of maintaining high degree of tissue colonisation (Figure $4 \mathrm{C}$ and D)

23 with the development of a significant vascular network hence avoiding a necrotic core. 24 Noteworthy, the scaffold did not show any sign of an acute degradation using this

25 subcutaneous model and the inflammatory response while still present did not increase 26 notably. 


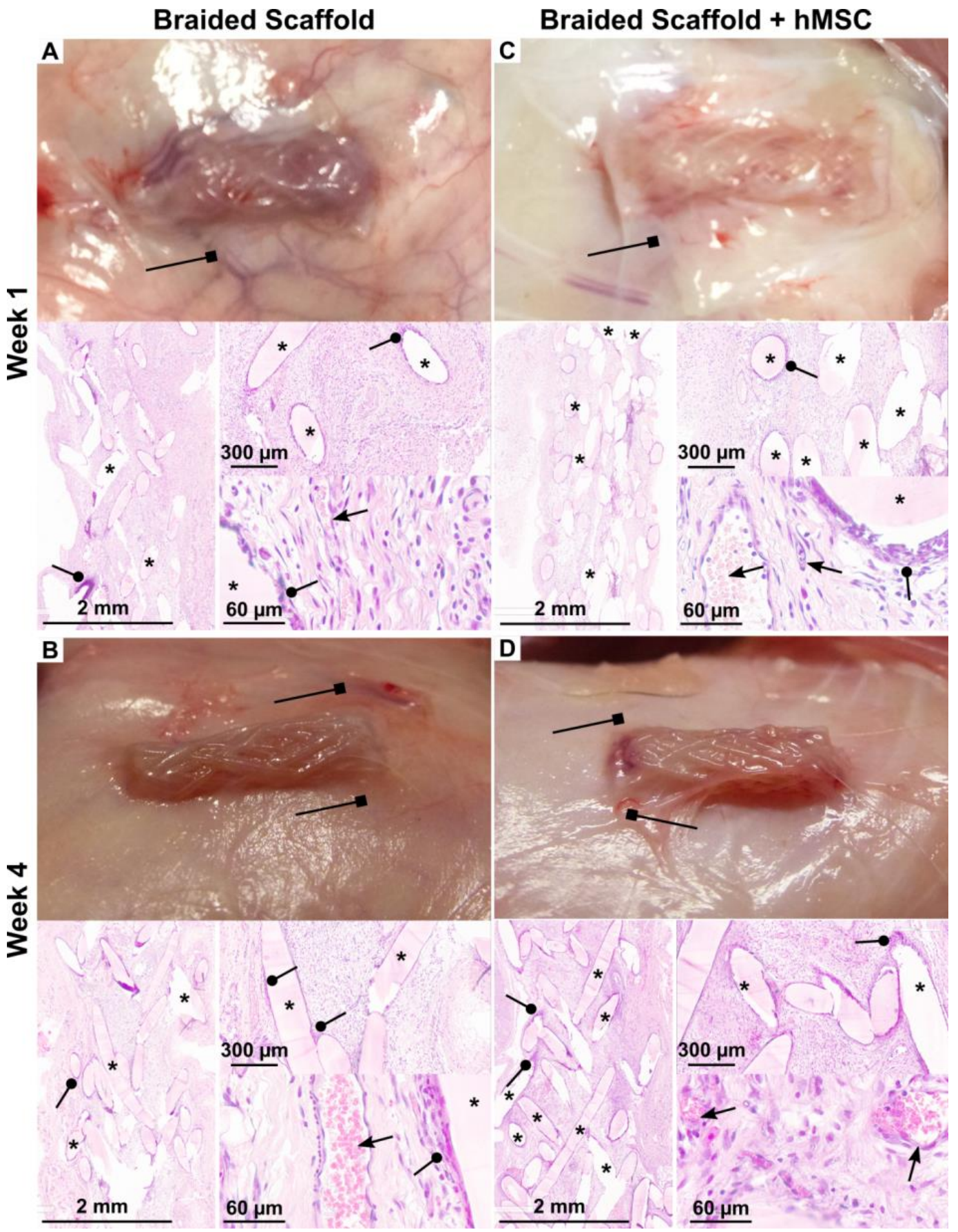

2 Figure 4: In vivo regenerative outcome of the braided scaffold using a subcutaneous 3 rodent model. A and C: Gross morphology of the control scaffold (no cells) with H\&E 4 staining revealing the tissue morphology 1 and 4 weeks post-implantation respectively, 5 indicating excellent tissue colonisation and vascularisation. B and D: Gross morphology 6 and H\&E staining and the cellularised braided scaffold 1 and 4 weeks post-implantation. $7 *$ denote the polymer filament and the void created by the fibres during the histology 8 process, Square arrow head indicates blood vessels near the scaffolds, round arrow head 9 indicates the presence of polynucleated cells, arrow indicates blood vessels and 10 capillaries in to core of the scaffold. 


\section{Effect of hydrolytic degradation on mechanical properties}

2 Typical experimental results at day 0,32 and 75 are reported in Figure 5. The decrease in

3 Young's modulus was clearly observed during the degradation process, as reported in 4 previous studies ${ }^{48}$. However, less intuitive behaviour was observed concerning the 5 inelastic part of the response. Firstly, the maximal admissible stress gradually increased

6 in the first month, and then decreased. Secondly, the strain to rupture gradually increased 7 in the first month until it reached high values (more than 50\%), and then drastically 8 decreased from the sixth week. Fibres were rather brittle at day 75 , with a strain to 9 rupture around $2 \%$.

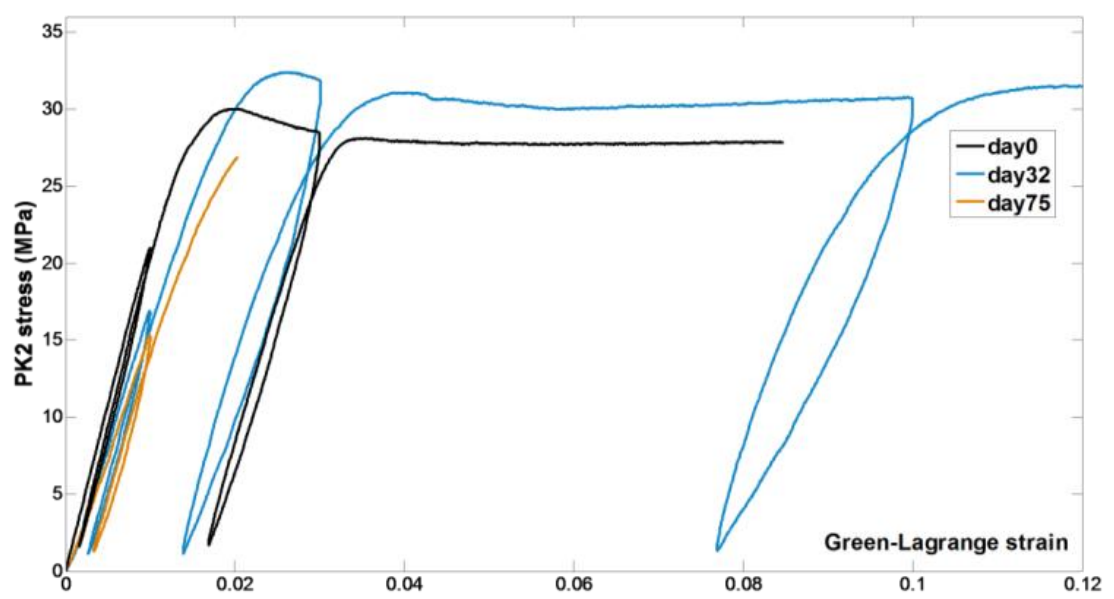

11 Figure 5 : Typical experimental results for individual fibres at day 0, 32 and 75.

12 The Chaboche-type elasto-plastic law was fitted to stress-strain curves for each tensile

13 test $(\mathrm{n}=4)$, for time steps equal to $0,5,8,14,19,26,32,40,49,54,60,67,75$ days. The 14 results in terms of the evolution of material parameters are illustrated in Figure 6. These 15 evolutions were simplified by phenomenological models in order to represent the global 16 tendency of the evolution of fibres properties during degradation. The Young's modulus 17 was represented using a damage variable as introduced in equation (5), and the fitting 18 algorithm resulted in the value $\mu^{E}=0.0024$. Given the experimental results for the 19 hardening parameter $c$, a similar law was used introducing a new decrease rate $\mu^{c}$ and a constant value $c_{\infty}$ such that the hardening parameter does not tend to zero. Fitted parameters were $\mu^{c}=0.6$ and $c_{\infty}=100$. Yield strain showed a non-monotonic evolution characterized by a slight initial increase followed by a decrease, such as it finally tended to its initial value. In the simulations, a constant value of $\varepsilon_{0}=0.1$ was selected because of the lack of physical basis describing such an evolution. No values of hardening parameter or yield strain were obtained from day 60 , as long as the fibres were brittle and failed 26 before entering the plastic region. 

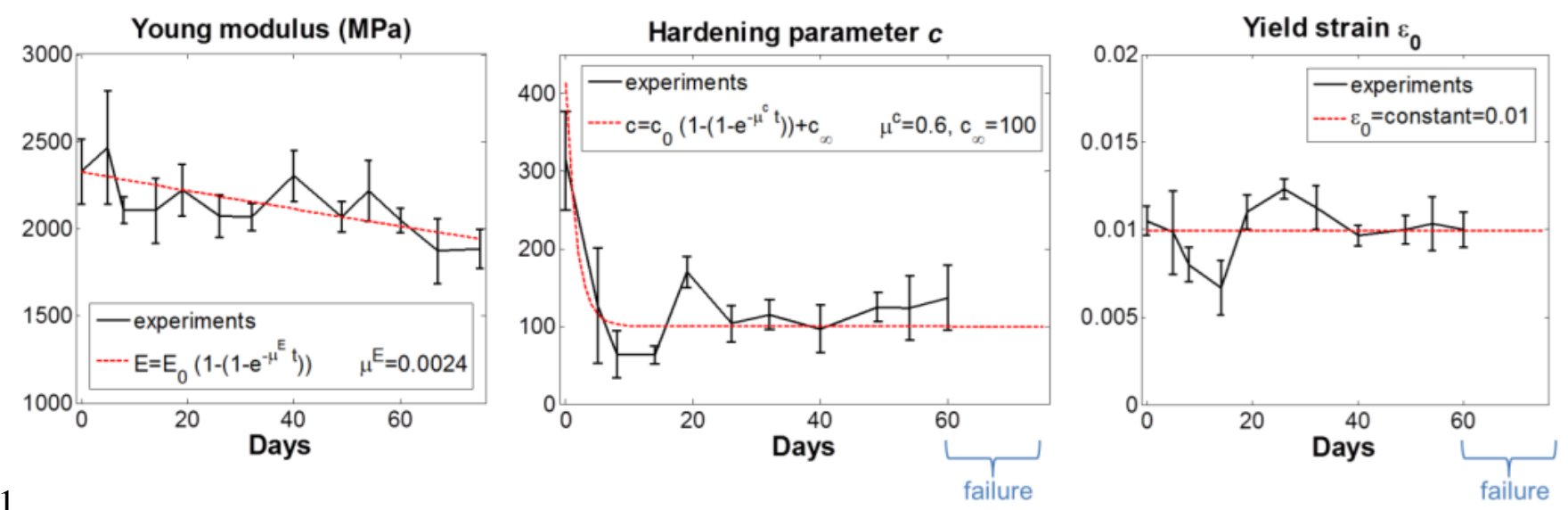

2 Figure 6 : Fitted material parameters of individual fibers during hydrolytic degradation.

3 The evolution of force-strain response of the braided scaffold due to polymer degradation

4 is represented in Figure 7. A decrease in elastic properties of the scaffold due to the

5 degradation of individual fibres was clearly observed. No accumulation of plastic strain

6 during the degradation was observed even after 6 months.
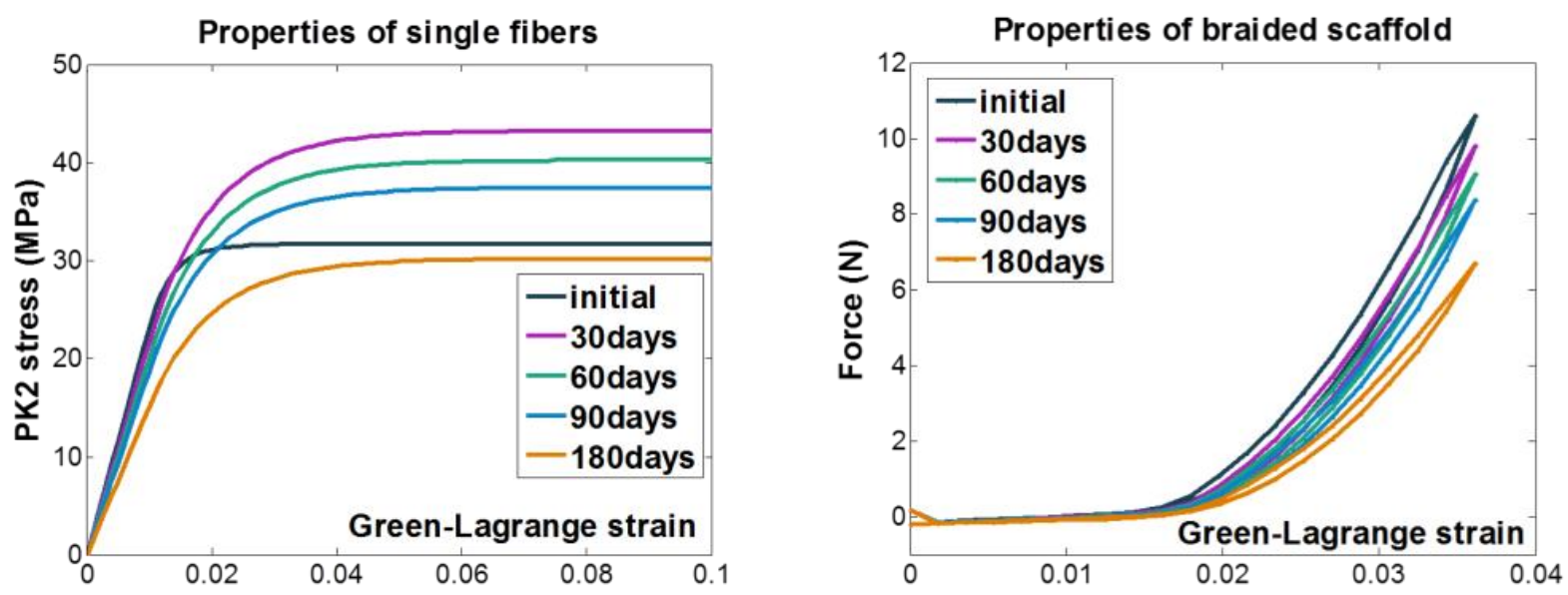

8 Figure 7 : Simulated evolution of single fibres and scaffold response.

9 Resulting from these data, we estimated the ideal contribution of tissue growth to the

10 evolution of construct mechanical properties, as represented in Figure 8. 
Properties of single fibers

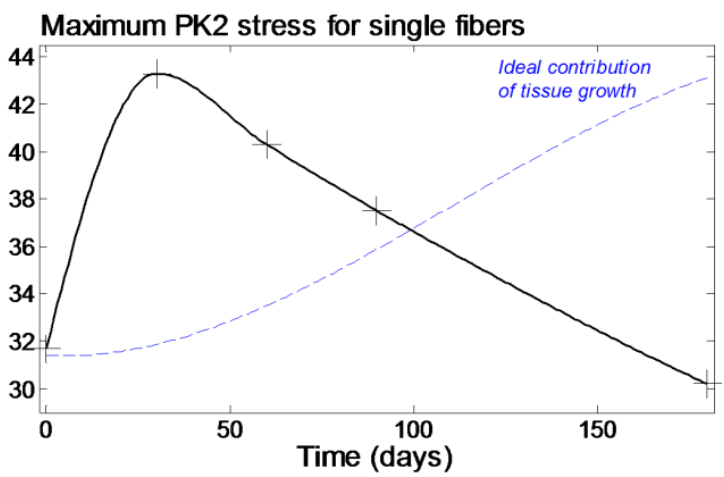

Properties of braided scaffold

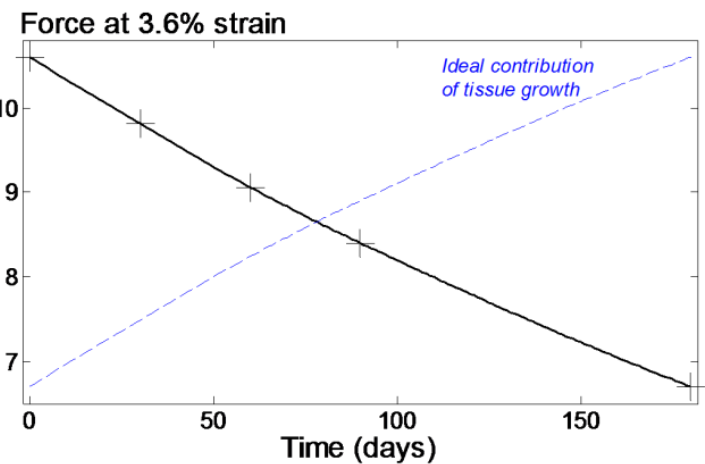

2 Figure 8: Ideal contribution of tissue growth to the evolution of the maximal stress (left)

3 and the force reached for a 3.6\% strain of the scaffold (right). The ideal contribution of

4 tissue growth is represented with a blue dashed line.

5 The distribution of OSS before and after squat exercise at each time step is represented in

6 Figure 9. At day 60, around 9\% of the OSS at the scaffold surface was above 2\%, which

7 corresponds to the strain at failure experimentally observed for individual fibres at this

8 time point. These maximum strains were observed in the central layers of the scaffold,

9 since the fibre recruitment during loading occured from the core to the periphery of the

10 scaffold.

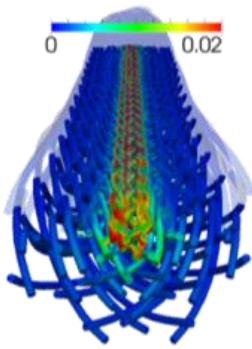

initial
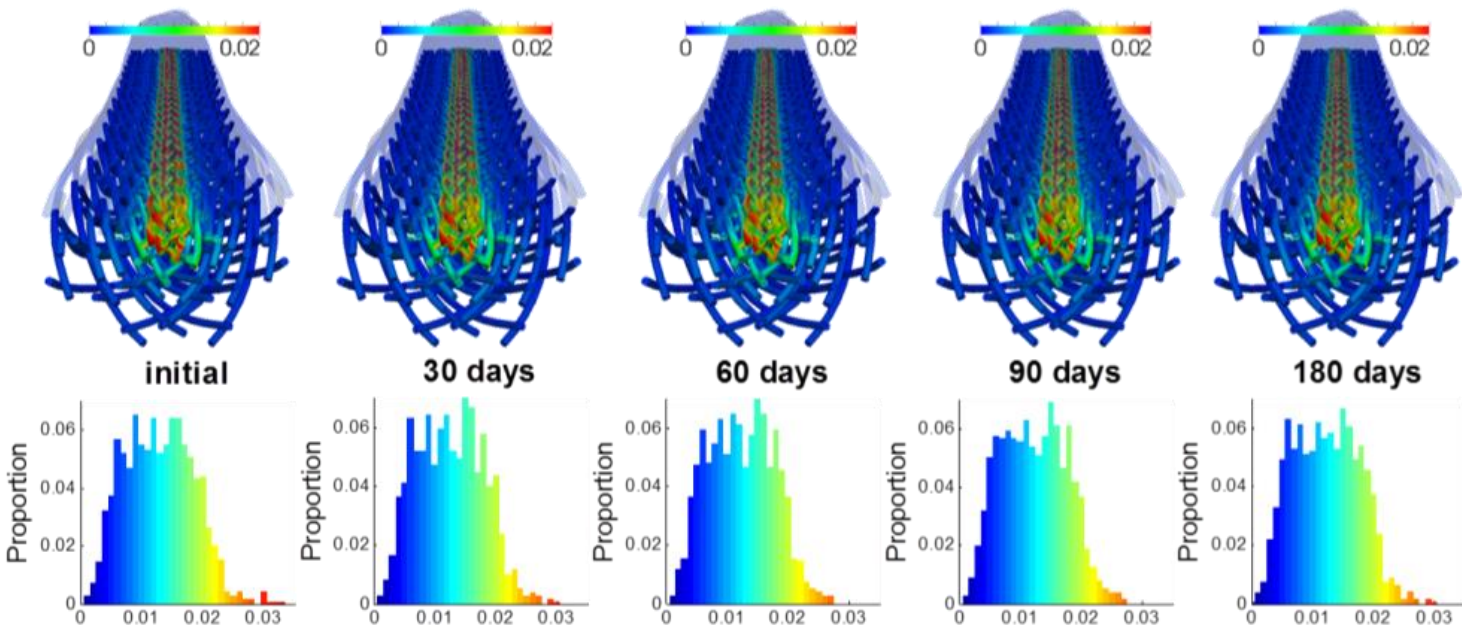

180 days
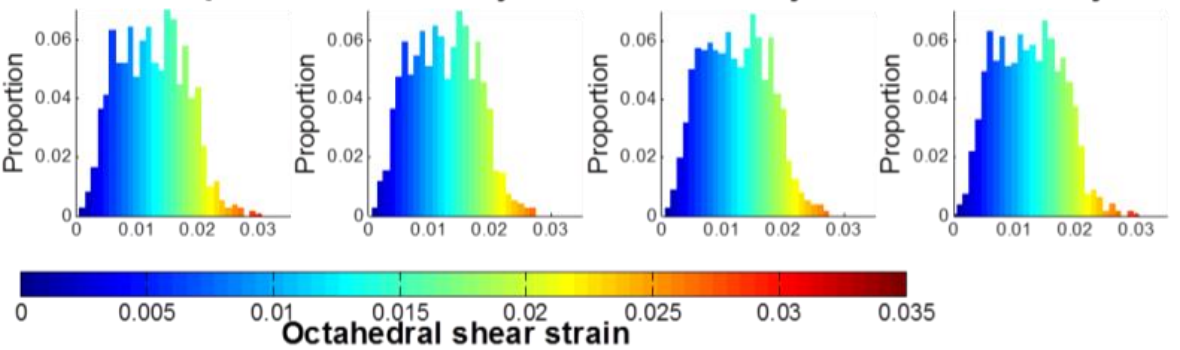

12 Figure 9 : Evolution of OSS distribution at the scaffold surface during scaffold

13 degradation. 
While the issue of choosing the adequate material for a given tissue engineering application is still a milestone, we proposed in the present contribution to provide new data concerning the mechanical and biological properties of a PLCL braided scaffold. A recent questionnaire has indeed shown that surgeons would consider using tissueengineered ligament "provided that it showed biological and mechanical success" 54 . Firstly, we reported the results of in vivo subcutaneous implantations of PLCL scaffolds using a rat model. From histological observations, the PLCL braided scaffold was shown to be suitable for tissue engineering since it promoted tissue colonization, even when initially non-cellularized, as well as external vascularization. No particular inflammatory response was observed, and no degradation was detected 4 weeks after implantation. No difference was observed between acellular or cellularised scaffold, indicating a high capability of maintaining high degree of tissue colonisation and vascularization for both groups.

15 We also reported additional data concerning the in vitro biocompatibility of PLCL scaffolds for two different types of cells that constitute privileged cell sources for soft tissue engineering. Satisfying results were obtained concerning the cell proliferation and collagen secretion for stem cells issued both bone marrow and Wharton jelly, indicating that BM-MSC-scaffold and WJ-MSC-scaffold both have a good potential to be used as biomaterial for tissue regeneration. No significant difference between these two types of cells were observed, while different responses of BM-MSC and WJ-MSC have been reported in the litterature ${ }^{38}$. The results indicated that WJ-MSC showed a high capacity to colonize the PLCL scaffold. As a result, WJ-MSC may constitute a promising alternative to BM-MSC due to their accessibility and expansion potential ${ }^{38}$. From these in vivo and in vitro studies, PLCL braided scaffolds appeared to be a suitable choice for various tissue engineering applications due to their excellent biocompatibility.

In a second part of this work, we reported original data concerning the effect of degradation on the elasto-plastic behaviour of PLCL fibers. Material parameters of a Chaboche-type elasto-plastic law were fitted from experimental data over more than two months. Irregular curves and substantial standard deviations were however obtained, and may be attributed to irregularities in fibre diameter (due to the custom extrusion process), and to the non-unicity of the fitted elasto-plastic law. These data were then extrapolated using a computational approach in order to assess the mechanical properties of the braided scaffold, and therefore its suitability for load-bearing tissue engineering applications. A typical example of Anterior Cruciate Ligament (ACL) tissue engineering was taken as one of those load-bearing applications. Interestingly, the elasto-plastic response of fibres changed in a non-intuitive way due to degradation, showing an increase in strain at failure and maximal stress followed by a sudden decrease of these parameters. This evolution differed from observations recently reported on a jet-printed PCL scaffold for tendon repair ${ }^{44}$. It therefore indicates that different materials and structures may have drastically different degradation mechanisms and profiles, and it emphasizes the need to systematically study this evolution before drawing conclusions about the suitability of a scaffold for a given application. A first element of explanation of such an evolution of material properties could be the shortening of polymeric chains 
due to hydrolysis, which then come small enough to act as a plastifier, hence increasing the stain at failure. On the other hand, as the chain were shortened during hydrolysis, they re-crystallisation could explain the observed increase in the stress at yield. To validate this assumption, further physico-chemical experiments may be conducted in the future on this particular polymer during hydrolytic degradation. It is worthy to note that the evolution of individual fibres mechanical properties was characterized based uniquely on hydrolytic degradation within distilled water at $37^{\circ} \mathrm{C}$. However, it has been previously reported that enzyme degradation occurring in vivo may catalyse the polymer degradation, and that in vivo and in vitro degradation may be heterogeneous ${ }^{55,56}$. In order to overcome this limitation, a large experimental study aiming at characterizing the properties of fibres (and/or braided scaffolds) after in vivo implantation could be performed in the future. Alternatively, the degradation of fibres could have been performed in a different solution, containing additives, enzymes or drugs.

The FE simulations then permitted to state that this degradation did not induced any accumulation of plastic strain within the scaffold for the physiological conditions of ACL. Due to structural effects, the scaffold did not enter into the plastic zone for the simulated physiological loading (3.6\% of global strain), even if the fibres would individually deform plastically for such a strain. After 6 months, the loading capacity of the scaffold decreased to around $40 \%$. This decrease should then ideally be compensated by tissue growth and the creation of a collagen network. Thanks to these simulation, the ideal participation of tissue growth to the maintain of suited mechanical properties was estimated. However, and more importantly, these results showed that the fibres exhibited a brittle behaviour after two months of in vitro degradation. The analysis of simulation results permitted to affirm that the central fibres of the scaffold may fail for postimplantation time above 60 days, since the local strains were superior to the observed strain at failure. Alternatively, it may be concluded that tissue growth should compensate this brittle behaviour within 60 days after implantation.

Several original conclusions may be drawn from the present study, answering gaps of knowledge present in the current state of art. Firstly, from a purely biological point of view, we confirmed that PLCL was a suitable choice for various tissue engineering applications, seeded or non-seeded with BM-MSC and WJ-MSC. Secondly, we emphasized that the mechanical properties of the selected material showed a non-intuitive evolution due to degradation. As a consequence, the conclusions that were made initially concerning the suitability of the PLCL scaffold used in this study for load-bearing applications such as ACL tissue engineering ${ }^{33}$ may not be valid anymore if we consider this evolution in time. In fact, we showed that the risk of brittle failure of PLCL scaffold was high after 2 months of degradation due to the change it strain at failure. It contradicts premature conclusions that have been drawn in the literature, including in our previous studies $^{33,35}$, concerning the adapted mechanical response of this scaffold. Consequently, it appears then crucial from these results that a sufficient tissue growth compensate this evolution of mechanical properties within a period of 2 months, which should be assessed in the future during long-term culture within the dedicated bioreactor developed in our team ${ }^{34}$. This study emphasizes the need to characterize the properties of biomaterials in a pluridisciplinary approach, and to consider the evolution of these mechanical properties prior to the assessment of their suitability for a given application. It is worthy to 46 emphasize that this study was conducted for a specific type of PLCL, with a PLLA/PCL 
1 ratio of 85/15. Different results may be obtained for different ratios, and therefore one

2 must not conclude too rapidly that PLCL is to be excluded for any soft tissue engineering 3 application in the future. Alternatively, work may be performed in the future in order to

4 optimize the PLCL chemical composition in such a way that the observed brittle

5 behaviour is reduced.

$6 \quad$ Funding

7 The author(s) received no financial support for the research, authorship, and/or 8 publication of this article.

9 References

10 1. Nair LS, Laurencin CT. Biodegradable polymers as biomaterials. Prog Polym Sci $112007 ; 32: 762-798$.

12 2. Manavitehrani I, Fathi A, Badr H, et al. Biomedical Applications of Biodegradable

13 Polyesters. Polymers 2016; 8: 20.

14 3. Goonoo N, Bhaw-Luximon A, Jhurry D. Biodegradable polymer blends: miscibility, 15 physicochemical properties and biological response of scaffolds. Polym Int 2015;

16 64: 1289-1302.

17 4. Sheikh Z, Najeeb S, Khurshid Z, et al. Biodegradable Materials for Bone Repair and 18 Tissue Engineering Applications. Materials 2015; 8: 5744-5794.

19 5. Hamad K, Kaseem M, Ko YG, et al. Biodegradable polymer blends and composites:

20 An overview. Polym Sci Ser A 2014; 56: 812-829.

21 6. Goonoo N, Bhaw-Luximon A, Bowlin GL, et al. An assessment of biopolymer- and synthetic polymer-based scaffolds for bone and vascular tissue engineering. Polym Int 2013; 62: 523-533.

247 . Cheung H-Y, Lau K-T, Lu T-P, et al. A critical review on polymer-based bioengineered materials for scaffold development. Compos Part B Eng 2007; 38: 291300 .

27 8. Eisenbarth E. Biomaterials for tissue engineering. Adv Eng Mater 2007; 9: 1051$28 \quad 1060$.

29 9. Vilay V, Mariatti M, Ahmad Z, et al. Characterization of the mechanical and thermal properties and morphological behavior of biodegradable poly(Llactide)/poly( $\varepsilon$-caprolactone) and poly(L-lactide)/poly(butylene succinate-co-Llactate) polymeric blends. J Appl Polym Sci 2009; 114: 1784-1792. 
10. Hiljanen-Vainio M, Karjalainen T, Seppälä J. Biodegradable lactone copolymers. I. Characterization and mechanical behavior of $\varepsilon$-caprolactone and lactide copolymers. J Appl Polym Sci 1996; 59: 1281-1288.

11. Vieira AC, Guedes RM, Marques AT. Development of ligament tissue biodegradable devices: A review. J Biomech 2009; 42: 2421-2430.

12. Hiljanen-Vainio MP, Orava PA, Seppälä JV. Properties of $\epsilon$-caprolactone/DLlactide ( $\epsilon-\mathrm{CL} / \mathrm{DL}-\mathrm{LA})$ copolymers with a minor $\epsilon-\mathrm{CL}$ content. J Biomed Mater Res 1997; 34: 39-46.

13. Lu XL, Cai W, Gao ZY. Shape-Memory Behaviors of Biodegradable Poly(Llactide-co- $\varepsilon$-caprolactone) Copolymers. J Appl Polym Sci 2008; 108: 1109-1115.

14. Vaquette C, Frochot C, Rahouadj R, et al. Mechanical and Biological characterization of A Porous Poly-L-Lactic Acid-Co- $\epsilon$-Caprolactone scaffold for Tissue Engineering. Soft Mater 2008; 6: 25-33.

15. Jung Y, Park MS, Lee JW, et al. Cartilage regeneration with highly-elastic threedimensional scaffolds prepared from biodegradable poly(l-lactide-co- $\varepsilon$ caprolactone). Biomaterials 2008; 29: 4630-4636.

16. Jeong SI, Kim B-S, Lee YM, et al. Morphology of Elastic Poly(l-lactide-co- $\varepsilon-$ caprolactone) Copolymers and in Vitro and in Vivo Degradation Behavior of Their Scaffolds. Biomacromolecules 2004; 5: 1303-1309.

17. Jeong SI, Kim B-S, Kang SW, et al. In vivo biocompatibilty and degradation behavior of elastic poly(1-lactide-co-e-caprolactone) scaffolds. Biomaterials 2004; 25: 5939-5946.

18. Pangesty AI, Arahira T, Todo M. Development and characterization of hybrid tubular structure of PLCL porous scaffold with hMSCs/ECs cell sheet. J Mater Sci Mater Med 2017; 28: 165.

19. Vaquette C, Kahn C, Frochot C, et al. Aligned poly(L-lactic-co-e-caprolactone) electrospun microfibers and knitted structure: A novel composite scaffold for ligament tissue engineering. J Biomed Mater Res A 2010; 94A: 1270-1282.

20. Lee J, Tae G, Kim YH, et al. The effect of gelatin incorporation into electrospun poly(l-lactide-co-e-caprolactone) fibers on mechanical properties and cytocompatibility. Biomaterials 2008; 29: 1872-1879.

21. Liu Z, Yu N, Holz FG, et al. Enhancement of retinal pigment epithelial culture characteristics and subretinal space tolerance of scaffolds with $200 \mathrm{~nm}$ fiber topography. Biomaterials 2014; 35: 2837-2850. 
22. Keun Kwon I, Kidoaki S, Matsuda T. Electrospun nano- to microfiber fabrics made of biodegradable copolyesters: structural characteristics, mechanical properties and cell adhesion potential. Biomaterials 2005; 26: 3929-3939.

23. Horakova J, Mikes P, Saman A, et al. Comprehensive assessment of electrospun scaffolds hemocompatibility. Mater Sci Eng C 2018; 82: 330-335.

24. Techaikool P, Daranarong D, Kongsuk J, et al. Effects of plasma treatment on biocompatibility of poly[(L-lactide)-co-( $\epsilon$-caprolactone $)]$ and poly[(L-lactide)-coglycolide] electrospun nanofibrous membranes. Polym Int 2017; 66: 1640-1650.

25. Inoguchi $\mathrm{H}, \mathrm{K}$ won IK, Inoue $\mathrm{E}$, et al. Mechanical responses of a compliant electrospun poly(l-lactide-co-\&-caprolactone) small-diameter vascular graft. Biomaterials 2006; 27: 1470-1478.

26. Daranarong D, Techaikool P, Intatue W, et al. Effect of surface modification of poly(l-lactide-co-e-caprolactone) membranes by low-pressure plasma on support cell biocompatibility. Surf Coat Technol 2016; 306, Part A: 328-335.

27. Wu C, An Q, Li D, et al. A novel heparin loaded poly(l-lactide-co-caprolactone) covered stent for aneurysm therapy. Mater Lett 2014; 116: 39-42.

28. Chen X, Wang J, An Q, et al. Electrospun poly(1-lactic acid-co- $\varepsilon$-caprolactone) fibers loaded with heparin and vascular endothelial growth factor to improve blood compatibility and endothelial progenitor cell proliferation. Colloids Surf B Biointerfaces 2015; 128: 106-114.

29. Kim SH, Kim SH, Jung Y. TGF- $\beta 3$ encapsulated PLCL scaffold by a supercritical CO2-HFIP co-solvent system for cartilage tissue engineering. J Controlled Release 2015; 206: 101-107.

30. Park JH, Hong JM, Ju YM, et al. A novel tissue-engineered trachea with a mechanical behavior similar to native trachea. Biomaterials 2015; 62: 106-115.

31. Jeong SI, Lee YM, Shin H. Tissue engineering using a cyclic strain bioreactor and gelatin/PLCL scaffolds. Macromol Res 2008; 16: 567-569.

32. Laurent CP, Ganghoffer J-F, Babin J, et al. Morphological characterization of a novel scaffold for anterior cruciate ligament tissue engineering. J Biomech Eng 2011; 133: 065001.

33. Laurent CP, Durville D, Mainard D, et al. A multilayer braided scaffold for Anterior Cruciate Ligament: Mechanical modeling at the fiber scale. J Mech Behav Biomed Mater 2012; 12: 184-196.

34. Laurent CP, Vaquette C, Martin C, et al. Towards a Tissue-Engineered Ligament: Design and Preliminary Evaluation of a Dedicated Multi-Chamber Tension-Torsion Bioreactor. Processes 2014; 2: 167-179. 
35. Laurent CP, Latil P, Durville D, et al. Mechanical behaviour of a fibrous scaffold for ligament tissue engineering: Finite elements analysis vs. X-ray tomography imaging. J Mech Behav Biomed Mater 2014; 40: 222-233.

36. Lee J, Choi WI, Tae G, et al. Enhanced regeneration of the ligament-bone interface using a poly(l-lactide-co-e-caprolactone) scaffold with local delivery of cells/BMP2 using a heparin-based hydrogel. Acta Biomater 2011; 7: 244-257.

37. Laurent CP, Durville D, Vaquette C, et al. Computer-Aided Tissue Engineering: application to the case of Anterior Cruciate Ligament Repair. In: Biomechanics of cells and tissues. 2013, pp. 1-44.

38. Prasanna SJ, Gopalakrishnan D, Shankar SR, et al. Pro-Inflammatory Cytokines, IFN $\gamma$ and $\mathrm{TNF} \alpha$, Influence Immune Properties of Human Bone Marrow and Wharton Jelly Mesenchymal Stem Cells Differentially. PLOS ONE 2010; 5: e9016.

39. Fernández J, Etxeberria A, Ugartemendia JM, et al. Effects of chain microstructures on mechanical behavior and aging of a poly(L-lactide-co--caprolactone) biomedical thermoplastic-elastomer. J Mech Behav Biomed Mater 2012; 12: 29-38.

40. Fernández J, Etxeberria A, Sarasua J-R. Synthesis, structure and properties of poly(L-lactide-co--caprolactone) statistical copolymers. J Mech Behav Biomed Mater 2012; 9: 100-112.

41. Chung EJ, Sugimoto MJ, Koh JL, et al. A biodegradable tri-component graft for anterior cruciate ligament reconstruction. J Tissue Eng Regen Med 2017; 11: 704712 .

42. Tainio J, Paakinaho K, Ahola N, et al. In Vitro Degradation of Borosilicate Bioactive Glass and Poly(l-lactide-co-e-caprolactone) Composite Scaffolds. Materials 2017; 10: 1274.

43. Petisco-Ferrero S, Etxeberria A, Sarasua JR. Mechanical properties and state of miscibility in poly(racD,L-lactide-co-glycolide)/(L-lactide-co-e-caprolactone) blends. J Mech Behav Biomed Mater 2017; 71: 372-382.

44. Wu Y, Wong YS, Fuh JYH. Degradation behaviors of geometric cues and mechanical properties in a 3D scaffold for tendon repair. J Biomed Mater Res A 2017; 105: 1138-1149.

45. Vieira AC, Guedes RM, Tita V. Damage-induced hydrolyses modelling of biodegradable polymers for tendons and ligaments repair. J Biomech 2015; 48: $3478-3485$.

46. Vieira AC, Guedes RM, Tita V. Constitutive modeling of biodegradable polymers: Hydrolytic degradation and time-dependent behavior. Int J Solids Struct 2014; 51: $1164-1174$. 
1 47. Vieira AC, Guedes RM, Tita V. Constitutive models for biodegradable thermoplastic ropes for ligament repair. Compos Struct 2012; 94: 3149-3159.

3 48. Vieira AC, Vieira JC, Ferra JM, et al. Mechanical study of PLA-PCL fibers during in vitro degradation. J Mech Behav Biomed Mater 2011; 4: 451-460.

49. Durville D. Contact-friction modeling within elastic beam assemblies: an application to knot tightening. Comput Mech 2012; 49: 687-707.

7 50. Lemaitre J, Chaboche J-L. Mechanics of Solid Materials. 1 edition. Cambridge:

8 Cambridge University Press, 1990.

9 51. Karmani S, Ember T. The anterior cruciate ligament-II. Curr Orthop 2004; 18:

$10 \quad 49-57$.

11 52. Beynnon BD, Fleming BC. Anterior cruciate ligament strain in-vivo: A review of 12 previous work. J Biomech 1998; 31: 519-525.

13 53. Lacroix D, Chateau A, Ginebra M-P, et al. Micro-finite element models of bone 14 tissue-engineering scaffolds. Biomaterials 2006; 27: 5326-5334.

15 54. Rathbone S, Maffulli N, Cartmell SH. Most British Surgeons Would Consider Using a Tissue-Engineered Anterior Cruciate Ligament: A Questionnaire Study. Stem Cells International. Epub ahead of print 2012. DOI: 10.1155/2012/303724.

18 55. Anderson JM, Shive MS. Biodegradation and biocompatibility of PLA and PLGA microspheres. Adv Drug Deliv Rev 2012; 64, Supplement: 72-82.

56. Dånmark S, Finne-Wistrand $\mathrm{A}$, Schander K, et al. In vitro and in vivo degradation profile of aliphatic polyesters subjected to electron beam sterilization. Acta Biomater 2011; 7: 2035-2046. 